\title{
A Quintessence Scalar Field in Brans-Dicke Theory
}

\author{
Narayan Banerjee円 and Diego Pavón円 \\ Departamento de Física. Facultad de Ciencias. Edificio Cc. \\ Universidad Autónoma de Barcelona. \\ E-08193 Bellaterra (Barcelona). Spain
}

\begin{abstract}
It is shown that a minimally coupled scalar field in Brans-Dicke theory yields a non-decelerated expansion for the present universe for open, flat and closed Friedmann-Robertson-Walker models.
\end{abstract}

PACS number: $9880 \mathrm{H}$

\section{Introduction}

The recent extensive search for a matter field which can give rise to an accelerated expansion for the universe stems from the observational data regarding the luminosity-redshift relation of the type Ia supernovae up to about $z \sim 1$ [四]. This matter field is called a "quintessence matter" (Q-matter for short). The most popular candidate as a Q-matter has so far been a scalar field having a potential which generates a sufficeint negative pressure at the present epoch [2]. Amongst the scalar fields considered as the Q-matter, the tracker field slowly rolling down its potential as proposed by Zlatev, Wang and Steinhardt [3] appears to be very promising. Some exotic matter like the domain walls or cosmic strings also find themselves amongst the possible candidates [4]. Unfortunately most of these fields work only for a spatially flat $(k=0)$ FRW model. Very recently, Chimento et al. [5] showed that a combination of dissipative effects like a bulk viscous stress and a quintessence scalar field gives an accelerated expansion for an open universe $(k=-1)$ as well. This model also provides a solution for the coincidence problem as the ratio of the density parameters corresponding to the normal matter and the quintessence field asymptotically approaches a constant value. Recently Bertolami and Martins [6] obtained an accelerated expansion for the universe in a modified Brans-Dicke (BD) theory by introducing a potential which is a function of the Brans-Dicke scalar field itself.

The present work investigates the possibility of obtaining a non-decelerating $\left(q \equiv-a \ddot{a} /(\dot{a})^{2} \leq 0\right)$ expansion for the universe in Brans-Dicke theory with the help of another scalar field which is minimally coupled to gravity and serves as the quintessence matter. Brans-Dicke theory played a major role in the attempts

\footnotetext{
${ }^{1}$ Permanent address: Relativity and Cosmology Research Centre, Department of Physics, Jadavpur University, Calcutta-700032, India

${ }^{2}$ Electronic mail address: diego@ulises.uab.es
} 
to solve the "graceful exit" problem in the inflationary scenario prevailing in the early universe. It is worthwhile to explore the possibilty of an application of the theory towards a reasonable explanation of the late time behaviour of the universe as well. We find that for a negative value of the Brans-Dicke parameter $\omega$, the theory leads to an accelerated expansion for the universe for a spatially flat model. The possible solutions contain the Bertolami-Martin's solution as well. Furthermore, the theory also leads to at least non-decelerating expansions even for non flat models. It should be noted that we do not modify Brans-Dicke theory by introducing a self interaction of the Brans-Dicke's scalar field, but rather look at the possibility of a non-decelerating solution with the help of a quintessence field within the purview of the theory itself.

\section{Field equations and the solutions}

The Brans - Dicke theory of gravity is given by the action

$$
S=\frac{1}{16 \pi G_{0}} \int \sqrt{-g}\left[\phi R-\omega \frac{\phi_{, \alpha} \phi^{, \alpha}}{\phi}+L_{m}\right] d^{4} x
$$

where $\phi$ is the BD scalar field, $\omega$ is the dimensionless constant BD parameter and $L_{m}$ is the Lagrangian for all other matter fields. If we assume the matter field to consist of a perfect fluid and a scalar field $\psi$ as the quintessence matter, the field equations for a Robertson-Walker spacetime are,

$$
\begin{gathered}
3 \frac{\left(\dot{a}^{2}+k\right)}{a^{2}}=\frac{\left(\rho_{m}+\rho_{\psi}\right)}{\phi}-3 \frac{\dot{a} \dot{\phi}}{a \phi}+\frac{\omega}{2} \frac{\dot{\phi}^{2}}{\phi^{2}}, \\
2 \frac{\ddot{a}}{a}+\frac{\left(\dot{a}^{2}+k\right)}{a^{2}}=\frac{-\left(p_{m}+p_{\psi}\right)}{\phi}-\frac{\omega}{2} \frac{\dot{\phi}^{2}}{\phi^{2}}-2 \frac{\dot{a} \dot{\phi}}{a \phi}-\frac{\ddot{\phi}}{\phi} .
\end{gathered}
$$

Here, $a$ is the scale factor of the Robertson-Walker metric, $k$ the spatial curvature index, $\rho_{m}$ and $p_{m}$ are the density and the pressure of the normal matter, $\rho_{\psi}$ and $p_{\psi}$ are those due to the quintessence field given by

$$
\rho_{\psi}=\frac{1}{2} \dot{\psi}^{2}+V(\psi), \quad p_{\psi}=\frac{1}{2} \dot{\psi}^{2}-V(\psi),
$$

where $V=V(\psi)$ is the relevant potential. The wave equation for the scalar field $\psi$ reads

$$
\ddot{\psi}+3 \frac{\dot{a}}{a} \dot{\psi}=-\frac{d V(\psi)}{d \psi}
$$

and the wave equation for the Brans-Dicke scalar field $\phi$ is

$$
\ddot{\phi}+\frac{3 \dot{a} \dot{\phi}}{a}=\frac{1}{2 \omega+3}\left[\left(\rho_{m}-3 p_{m}\right)+\left(\rho_{\psi}-3 p_{\psi}\right)\right] .
$$


The matter conservation equation,

$$
\dot{\rho_{m}}+3 \frac{\dot{a}}{a}\left(\rho_{m}+p_{m}\right)=0
$$

follows from the field equations. Assuming that at the present epoch the universe is filled with cold matter with negligible pressure, we put $p_{m}=0$, and this equation integrates to

$$
\rho_{m}=\rho_{1} / a^{3}
$$

where $\rho_{1}$ is an integration constant. Since our principal interest is to find an accelerating power law solution for the scale factor $a$, we shall assume that both $a$ and $\phi$ are power functions of the cosmic time $t$ in the form

$$
a=a_{1} t^{\alpha}, \quad \phi=\phi_{1} t^{\beta} .
$$

We shall look at the possibilities of consistent solutions with $a_{1}, \phi_{1}, \alpha, \beta$ are constants with $a_{1}, \phi_{1}$ being positve definite and $\alpha \geq 1$. These constants will be related amongst themselves and the characteristic constants of the theory through the field equations.

By combining ( 2) and ( 3) with (9) yields the expression for $\dot{\psi}^{2}$ as

$$
\dot{\psi}^{2}=\frac{2 k \phi_{1}}{a_{1}^{2}} t^{\beta-2 \alpha}+\left(2 \alpha+\alpha \beta-\omega \beta^{2}-\beta^{2}+\beta\right) \phi_{1} t^{\beta-2}-\frac{\rho_{1}}{a_{1}^{3}} t^{-3 \alpha} .
$$

The potential $V$ can be found out from the equation ( 6) as

$$
\begin{gathered}
V=-\frac{1}{2} \frac{\rho_{1}}{a_{1}^{3}} t^{-3 \alpha}+\frac{1}{2} \frac{k \phi_{1}}{a_{1}^{2}} t^{\beta-2 \alpha}+\frac{1}{4} \phi_{1}[(2 \omega+3)(\beta+3 \alpha-1) \beta \\
\left.+\left(2 \alpha+\alpha \beta-\omega \beta^{2}-\beta^{2}+\beta\right)\right] t^{\beta-2} .
\end{gathered}
$$

The wave equation for the quintessence scalar field $\psi$ ( 5), when multiplied by $\dot{\psi}$, looks like

$$
-\frac{d V}{d t}=\dot{\psi} \ddot{\psi}+3 \frac{\dot{a}}{a} \dot{\psi}^{2}
$$

which readily yields a first integral and hence an expression for $V$ as

$$
\begin{gathered}
V=-\frac{1}{2} \frac{\rho_{1}}{a_{1}^{3}} t^{-3 \alpha}-\frac{k \phi_{1}}{a_{1}^{2}} \frac{(\beta+4 \alpha)}{(\beta-2 \alpha)} t^{\beta-2 \alpha} \\
-\left(2 \alpha+\alpha \beta-\omega \beta^{2}-\beta^{2}+\beta\right) \frac{(\beta-2+6 \alpha)}{2(\beta-2)} \phi_{1} t^{\beta-2} .
\end{gathered}
$$

Now we demand that the right hand sides of ( 11) and ( 13) coincide. This leads to the required consistency relations amongst the constants. These relations 
lead to quite a few possibilities of solutions for an accelerating universe $(\alpha \geq 1)$. These possibilities fall into two broad classes, one in which $k=0$ and the second where $k$ is different from zero.

Case $1: k=0$

In this case, the consistency condition is

$$
6\left(4 \alpha^{2}-2 \alpha+2 \alpha^{2} \beta-\alpha \beta\right)=\omega \beta\left(\beta^{2}+6 \alpha \beta+12 \alpha-4\right) .
$$

It is easily seen that the condition ( 14) is automatically satisfied if $\beta=-2$, and so equation ( 10) reduces to

$$
\dot{\psi}^{2}=-\frac{2(2 \omega+3) \phi_{1}}{t^{4}}-\frac{\rho_{1}}{a_{1}^{3}} t^{-3 \alpha} .
$$

This indicates that $\omega<-3 / 2$ as $\dot{\psi}^{2}$ cannot be negative. For $\alpha=4 / 3$, one has $2|2 \omega+3| \phi_{1} \geq \frac{\rho_{1}}{a_{1}^{3}}$. In this case equation ( 15) integrates to

$$
\psi= \pm \frac{A}{t}
$$

where $A^{2}=-2(2 \omega+3) \phi_{1}-\frac{\rho_{1}}{a_{1}^{3}}>0$, and a simple form of $V(\psi)$ can be obtained,

$$
V=V_{1} \psi^{4}
$$

$V_{1}$ being a constant, related to the other constants of integrations like $a_{1}, \rho_{1}$ etc. through the field equations. With $\alpha=4 / 3$, deceleration parameter $q=-1 / 4$ and the model works for all time $0<t<\infty$ provided the condition $2|2 \omega+3| \phi_{1} \geq \rho_{1} / a_{1}^{3}$ is satisfied. The value of $\omega$ is related to the other constants through the relation

$$
2 \omega+3=-\frac{\rho_{1}}{a_{1}^{3} \phi_{1}} \pm \sqrt{\frac{2 a_{1}^{2} \phi_{1}-3 \rho_{1}}{6 a_{1} V_{1} \phi_{1}^{2}}} .
$$

For other values of $\alpha$ the model does not work for the whole range of time $0<t<\infty$. If $\alpha>4 / 3$, when the rate of acceleration is faster than $q=-1 / 4$, the model works only for $t>\left[\frac{\rho_{1}}{2 a_{1}^{3} \phi_{1}|2 \omega+3|}\right]^{\frac{1}{3 \alpha-4}}$. For $1<\alpha<4 / 3$, the model is valid only up to a time $t=\left[\frac{\rho_{1}}{2 a_{1}^{3} \phi_{1}|2 \omega+3|}\right]^{\frac{1}{3 \alpha-4}}$ during which the universe expands with an acceleration with a rate less than $q=-1 / 4$.

For $q=-1 / 4$, where the model works for the entire time span, the present age of the universe can be calculated from (2) as

$$
t_{0}=2 \sqrt{2}\left[\frac{(2 \omega+3)-\frac{\rho_{0}}{2 a_{0}^{3} \phi_{0}}-V_{1} A^{4}}{3(3 \omega+4)}\right]^{1 / 2} \frac{1}{H_{0}}
$$


In the large $\omega$ limit, this equation reduces to

$$
t_{0} \simeq \frac{8 \sqrt{-2 \omega V_{1}}}{3 H_{0}}
$$

where $\omega$ is obviously a negative quantity. Choosing $V_{1}=\frac{-9}{128} \omega^{-1}$ and $H_{0} \simeq 65$ $\mathrm{km} \cdot \mathrm{s}^{-1} / \mathrm{Mpc}$, the present age of the Universe turns out to be approximately 15 Gyr.

Likewise, the present rate of variation of $G$ is $|\dot{G} / G|_{0}=|\dot{\phi} / \phi|_{0}=\frac{3}{2} H_{0}<10^{-10}$ per year. This is quite compatible with the observational data (see [7] and references therein).

All these possibilities are for $\beta=-2$. For other values of $\beta$ also, one can obtain consistent solutions. Such as if $\beta=-1$, the equation ( 14) yields two possible solutions for $\alpha$, namely $1 / 2$ and $-\omega / 2$. We disregard $\alpha=1 / 2$ as we are interested in non-decelerating models where $\alpha \geq 1$. For $\omega=-2$, we have $\alpha=1$, i.e. an uniformly expanding universe with $q=0$. In this case, $\phi_{1}$ should be greater than $\rho_{1} / a_{1}^{3}$. The equation system can be easily solved to get $\psi \propto t^{-1 / 2}$ and $V \propto \psi^{6}$. In this uniformly expanding scenario, the model can work for the whole range of $0<t<\infty$. If $\omega$ is further negative, i.e., $\omega \leq-2$, we get faster rates of acceleration, but the model does not work for the whole range of time.

Case $2: k \neq 0$

In this case the condition ( 14) remains in place and a further condition from (11) and (13) is

$$
\beta=-2 \alpha
$$

It can be seen from the field equations ( 2) and ( 3) that one can obtain consistent solutions only for $\alpha=1$. So equation (20) immediately yields $\beta=-2$. With this value of $\beta$, the condition ( 14) is automatically satisfied for all values of $\omega$. In this case also the model works for a limited period of time, $0<t<t_{1}$, where

$$
t_{1}=2 \phi_{1}\left[\frac{k}{a_{1}^{2}}-(2 \omega+3)\right] \frac{a_{1}^{3}}{\rho_{1}} .
$$

For an open universe, i.e. for $k=-1,(2 \omega+3)$ has to be negative and $|2 \omega+3|>1 / a_{1}^{2}$.

For a closed universe $(k=1)$, the model works even for a positive $(2 \omega+3)$ provided $(2 \omega+3) a_{1}^{2}<1$. If however, $(2 \omega+3)$ is negative, the model holds good without any such relation between $a_{1}$ and $\omega$.

Thus, unlike most of proposed models with a non-positive definite deceleration 
parameter, a quintessence field in Brans-Dicke theory works for a spatially nonflat Robertson Walker spacetime as well. It is true that for $k \neq 0$ cases the only consistent solutions have $q=0$, but it is anyway non-decelerating and thus the expansion rate is faster than $t^{2 / 3}$, which might sufficiently explain the recent observations on the distant supernovae $[8]$.

It is worthy of note that the field equations can be integrated to produce consistent solutions for other forms of normal matter. Such as for a radiation dominated universe $\left(p_{m}=\rho_{m} / 3\right)$, a simple choice of $V$ as $V=V_{0} \psi^{6}$ and a negative $\omega$ will lead to a decelerated expansion $(q>0)$ for the universe with $a \propto t^{\frac{3}{4}}$ and $\phi \propto t^{-1}$. Thus the model can be interpolated back to earlier epoch to yield a decelerated universe which is required in order to explain processes like nucleosynthesis.

\section{A possible solution to the flatness problem}

One important aspect of this model is that potentially it can solve the flatness problem as well. To see this we effect a conformal transformation as

$$
\bar{g}_{\mu \nu}=\phi g_{\mu \nu},
$$

which enables us to identify the energy contributions from different components of matter very clearly. Equation (2), in this new version, looks like

$$
\frac{3\left(\dot{\bar{a}}^{2}+k\right)}{\bar{a}^{2}}=\bar{\rho}_{m}+\bar{\rho}_{\phi}+\bar{\rho}_{\psi},
$$

where a bar indicates quantities in the transformed version; $\rho_{m}, \rho_{\phi}, \rho_{\psi}$ are the contributions to the energy density from the normal matter, the BD scalar field and the quintessence scalar field respectively. The quantity $\bar{\rho}_{\phi}$ is actually given by

$$
\bar{\rho}_{\phi}=\frac{(2 \omega+3)}{4}\left(\frac{\dot{\phi}}{\phi}\right)^{2}=\bar{p}_{\phi} .
$$

The quantities describing the matter distribution are transformed as $\bar{\rho}_{i}=$ $\phi^{-2} \rho_{i}$ and $\bar{p}_{i}=\phi^{-2} p_{i}$. Now we define the dimensionless density parameter $\bar{\Omega}$ as

$$
\bar{\Omega}=\frac{\bar{\rho}}{3 \bar{H}^{2}}=\bar{\Omega}_{m}+\bar{\Omega}_{\phi}+\bar{\Omega}_{\psi},
$$

where the total density $\bar{\rho}=\bar{\rho}_{m}+\bar{\rho}_{\phi}+\bar{\rho}_{\psi}$, and the individual density paramaters $\bar{\Omega}_{i}$ are defined accordingly. Using the equation ( 231) and the equation for the conservation for the total energy,

$$
\dot{\bar{\rho}}+3 \dot{\bar{H}}(\rho+p)=0,
$$


we can write down the evolution equation for the density parameter as

$$
\dot{\bar{\Omega}}(\bar{\Omega}-1)(3 \gamma-2) \bar{H}=0
$$

The net barotropic index $\gamma$ is defined as

$$
\gamma \bar{\Omega}=\gamma_{m} \bar{\Omega}_{m}+\gamma_{\phi} \bar{\Omega}_{\phi}+\gamma_{\psi} \bar{\Omega}_{\psi}
$$

The individual $\gamma_{i}^{\prime} s$ are defined by the equation $p_{i}=\left(\gamma_{i}-1\right) \rho_{i}$. The ratioes $p_{i} / \rho_{i}$ remain the same in both frames and thus $\gamma_{i}$ 's do not vary. For our choice of matter, $\bar{p}_{m}=0$ and $\bar{p}_{\phi}=\bar{\rho}_{\phi}$, and thus $\gamma_{m}=1$ and $\gamma_{\phi}=2$. The third index $\gamma_{\psi}$, however, is not a constant and evolves with time via the equation $\gamma_{\psi}=\left(p_{\psi}+\rho_{\psi}\right) /\left(\rho_{\psi}\right)=\dot{\psi}^{2} /\left(\frac{1}{2} \dot{\psi}^{2}+V\right)$.

Equation ( 27) indicates that $\bar{\Omega}=1$ is indeed a solution. The stability of this solution demands that $(\partial \dot{\bar{\Omega}} / \partial \bar{\Omega})_{H}$ should be negative at $\bar{\Omega}=1$. Equation $(27)$ shows that for an expanding universe $(\bar{H}>0)$ this is possible only if $\gamma<2 / 3$. From equation (28) it can be shown that the relevant condition for $\gamma<2 / 3$ is

$$
\bar{\Omega}_{m}+4 \bar{\Omega}_{\phi}<\left(2-3 \gamma_{\psi}\right) \bar{\Omega}_{\psi},
$$

which can be achieved by a suitable adjustment of the parameters.

It is wellknown that geodesic equations are not valid in this conformally transformed version [9] and hence different quantities are not dependable regarding the content of their physical meaning. But it must be emphasized that the character of $k$ remains unaltered, and thus if $\Omega_{k}=k / a^{2}$ is zero in one frame, it must be so in the other as well.

\section{Concluding remarks}

A quintessence scalar field in Brans-Dicke theory is shown to give rise to an accelerated expansion for the present universe. Bertolami and Martins [6] modified Brans-Dicke theory by introducing a potential function $V=V(\phi)$ where $\phi$ is the Brans-Dicke scalar field. As Brans-Dicke theory by itself is, in a sense, selfinteracting (the kinetic term in the action contains $\phi$ ), we do not include such a potential. Rather a non-gravitational field $\psi$ with a potential $V=V(\psi)$ is included. For a spatially flat universe, the model yields various non-decelerating solutions including a uniformly expanding solution $(q=0)$. For a simple choice of the potential $\left(V \propto \psi^{4}\right)$, the model gives the solution of [6]. In this last case, the model can work for all time $0<t<\infty$. In most of the other accelerating solutions, the model is seen to work for only a restricted period of time.

An important merit of this ansatz is that it provides solutions for a non flat 
$(k \neq 0)$ Robertson Walker metric as well. Although these solutions are not accelerating, they are not decelerating either $(q=0)$. So along with providing a non-decelerating solution, it can potentially solve the flatness problem too. In fact it has been shown that $\Omega=1$ could be a stable solution in this model.

This acceleration of the universe is achieved, in general, by a negative $\omega$. It has been claimed that the value of $\omega$ should be large (> 500) if Brans-Dicke theory has to be consistent with the astronomical observations [10]. But this value actually refers to the magnitude of $\omega$ which can still be very large in this model except in some cases, such as for $(k=0, q=0)$, where $\omega=-2$. Furthermore, reconciliation with Kaluza-Klein theory or low-energy string theory favours a negative value of $\omega$ (see e.g. [11]).

\section{Acknowledgements}

This work has been partially supported by Spanish Ministry of Education under Grant PB94-0781. One of us (NB) is grateful to the "Direcció General de la Recerca" of the Catalonian Government for financial support under grant PIV99. The authors are grateful to Winfried Zimdahl for critically reading an earlier draft of this paper and useful suggestions.

\section{References}

[1] Perlmutter S et al. 1999 Astrophys. J. 517 565; Riess AG et al. 1998 Astron. J. 116 1009; Garnavich PM et al. 1998 Astrophys. J 50974

[2] Caldwell RR, Dave R and Steinhardt PJ 1998 Phys. Rev. Lett. 80 1582; Turner MS and White M 1997 Phys. Rev. D 56 R4439

[3] Zlatev I, Wang L and Steinhardt PJ 1999 Phys. Rev. Lett. 82 896; P.J. Steinhardt, L. Wang and I. Zlatev 1999 Phys. Rev. D 59, 123504

[4] Bucher M and Spergel D 1999 Phys. Rev. D 60 043505; Bucher M 1999 astro-ph/9908047

[5] Chimento LP, Jakubi AS and Pavón D 2000 Phys Rev. D (in the press) astro-ph/0005070

[6] Bertolami O and Martins PJ 2000 Phys. Rev. D 61064007

[7] Weinberg S 1972 Gravitation and Cosmology (New York: Wiley)

[8] Riess AG 2000 "The case for an accelerating universe from supernovae" astro-ph/0005229. 
[9] Dicke RH 1962 Phys. Rev. 1252163

[10] Will CM 1993 Theory and Experiment in Gravitational Physics (Cambridge: Cambridge University Press, 3rd edition)

[11] Kolitch SJ and Eardley DM 1995 Ann. Phys. (N.Y.) 241128 